\title{
Equilibrium and non-equilibrium effects in nucleus-nucleus collisions
}

\author{
L.V. Bravina a ${ }^{\mathrm{a}, \mathrm{b}, 2}$, M.I. Gorenstein ${ }^{\mathrm{a}, \mathrm{c}}$, E.E. Zabrodin ${ }^{\text {b,d }}$, S.A. Bass ${ }^{\mathrm{e}, 3}$, \\ M. Belkacem ${ }^{\mathrm{a}, 1}$, M. Bleicher ${ }^{\text {a }}$, M. Brandstetter ${ }^{\mathrm{a}}$, M. Hofmann ${ }^{\mathrm{a}}$, S. Soff ${ }^{\mathrm{a}, \mathrm{f}}$, \\ C. Spieles ${ }^{\text {g,3 }}$, H. Weber ${ }^{\text {a }}$, H. Stöckerand ${ }^{\text {a }}$, W. Greiner ${ }^{\text {a }}$ \\ ${ }^{a}$ Institut für Theoretische Physik, Goethe Universität Frankfurt, Germany \\ ${ }^{\mathrm{b}}$ Institute for Nuclear Physics, Moscow State University, 119899 Moscow, Russia \\ ${ }^{\mathrm{c}}$ Bogolyubov Institute for Theoretical Physics, Kiev, Ukraine \\ ${ }^{\mathrm{d}}$ Institut für Theoretische Physik, Universität Tübingen, Germany \\ ${ }^{\mathrm{e}}$ Dept. of Physics, Duke University, Durham, USA \\ ${ }^{\mathrm{f}}$ Gesellschaft für Schwerionenforschung, Darmstadt, Germany \\ g Nuclear Science Division, LBNL, Berkeley, CA 94720, USA \\ Received 19 March 1999; received in revised form 28 May 1999 \\ Editor: P.V. Landshoff
}

\begin{abstract}
Local thermal and chemical equilibration is studied for central A + A collisions at 10.7-160 AGeV in the Ultrarelativistic Quantum Molecular Dynamics model (UrQMD). The UrQMD model exhibits strong deviations from local equilibrium at the high density hadron-string phase formed during the early stage of the collision. Equilibration of the hadron-resonance matter is established in the central cell of volume $V=125 \mathrm{fm}^{3}$ at later stages, $t \geq 10 \mathrm{fm} / c$, of the resulting quasi-isentropic expansion. The thermodynamical functions in the cell and their time evolution are presented. Deviations of the UrQMD quasi-equilibrium state from the statistical mechanics equilibrium are found. They increase with energy per baryon and lead to a strong enhancement of the pion number density as compared to statistical mechanics estimates at SPS energies. (C) 1999 Published by Elsevier Science B.V. All rights reserved.
\end{abstract}

PACS: 25.75.-q; 24.10.Lx; 24.10.Pa; 64.30.+t

Keywords: Heavy ion collisions; QMD model; Local thermodynamical equilibrium; Equation of state; Non-equilibrium effects

\section{Introduction}

The main goal of relativistic heavy ion experiments at AGS Brookhaven and SPS CERN is to

\footnotetext{
${ }^{1}$ Alexander von Humboldt Foundation Fellow.

2 E-mail: bravina@th.physik.uni-frankfurt.de

${ }^{3}$ Feodor Lynen Fellow of the Alexander von Humboldt Foundation.
}

study the properties of strongly interacting hot and dense matter. The description of high energy nuclear (as well as hadronic) collisions may be achieved today only in terms of phenomenological macroscopic and microscopic models. Macroscopic models, like the well-known thermal [1-3] (see also [4-7]) and hydrodynamic [8,9] (see also [10-13]) models use the energy density $\varepsilon$, net baryon number density $\rho_{B}$ and pressure $P$ to describe the local 
properties of a system. To complete the set of hydrodynamic equations one postulates local thermodynamical equilibrium (LTE) and employs the equation of state (EoS) $P=P\left(\varepsilon, \rho_{B}\right)$ as driving term. Ideal fluid dynamics then leads to the entropy conservation law expressed in the form $s / \rho_{B}=$ const ( $s=s\left(\varepsilon, \rho_{B}\right)$ is the entropy density). The applicability of LTE concepts to nucleus-nucleus collisions $(\mathrm{A}+\mathrm{A})$ remains an ad hoc assumption which should be justified by microscopic dynamical model analysis. The question is still open despite of the intensive investigations (e.g. [14-17] and references therein).

In contrast to the hydrodynamic approach, the various Monte Carlo microscopic hadron-, string-, parton-cascade models [18-22] are based on assumptions about the interaction mechanisms between the constituents. No prerequisites of LTE are introduced. Microscopic models of nucleus-nucleus collisions exhibit, however, a huge number of elastic and inelastic rescatterings. For example, $\sim 1200$ baryonbaryon, $~ 3400$ meson-baryon and $\sim 2500$ mesonmeson collisions are found for $\mathrm{Pb}+\mathrm{Pb}$ at $160 \mathrm{AGeV}$ [23]. Elastic collisions should drive the system to local thermal equilibrium whereas inelastic collisions drive the chemical equilibration. The hydrodynamical picture of $\mathrm{A}+\mathrm{A}$ collisions can emerge from microscopic models. Thus, the approach to the LTE must be checked (and the EoS at different stages of $\mathrm{A}+\mathrm{A}$ collisions extracted) by means of microscopic models. Here, we employ for such analysis the Ultrarelativistic Quantum Molecular Dynamics (UrQMD) model [18], which reproduces nicely experimental spectra for mesons and baryons from 10 to 200 AGeV. Hadron matter equilibration has been studied with UrQMD simulations in a box with periodic boundary conditions in [24]. Results of simulations of heavy ion collisions were reported for $\mathrm{Au}+\mathrm{Au}$ at 10.7 AGeV in [25], see also [26] for lower energies. The present paper extends the analysis of relativistic heavy ion collisions to the energy region between AGS and SPS. New observable effects caused by the increase of the energy per baryon are predicted.

\section{UrQMD analysis of $A+A$ collisions}

Here, UrQMD model simulations are performed and analyzed for central (impact parameter zero, $b=0) \mathrm{Au}+\mathrm{Au}$ collisions at energy $10.7 \mathrm{AGeV}$ and $\mathrm{Pb}+\mathrm{Pb}$ collisions at $40 \mathrm{AGeV}$ and $160 \mathrm{AGeV}$. We briefly call them AGS, 'CERN', and SPS, respectively. Note that first experiments on $\mathrm{Pb}+\mathrm{Pb}$ collisions at $40 \mathrm{AGeV}$ have been done in CERN during the 1998 fall run and will be extended in 1999 [27]. The formation of hot and dense matter expected in these reactions is studied here. We fix a cubic cell of volume $V=5 \times 5 \times 5 \mathrm{fm}^{3}$ in the geometrical center of the $\mathrm{A}+\mathrm{A}$ system. The total momentum of this cell is close to zero during the whole time evolution of the $\mathrm{A}+\mathrm{A}$ reaction. The geometry of the system yields no preferable direction for the collective motion. The cell size should be small enough to avoid large collective flows inside the cell during the expansion stage. On the other hand it should be large enough to have sufficiently many particles inside the cell to guarantee reasonably small fluctuations for particle observables in the UrQMD simulations and reasonably small finite-size effects in the statistical model analysis. We fix the same cell size, $5 \times 5 \times 5$ $\mathrm{fm}^{3}$, which satisfies simultaneously both above requirements for all three reactions, at least for times $t \geq 2.5 \mathrm{fm} / c$ after contact, when the fastest pre-equilibrium particles will have left the scene.

We start by analyzing the time evolution for different physical quantities of the cell in the center of mass frame of $\mathrm{A}+\mathrm{A}$, which is also roughly because of the fluctuations, the proper rest frame of the cell considered. Time $t=0$ corresponds to the moment when the two Lorentz contracted nuclei just touch each other. The maximum overlap for the freely streaming nuclei is expected roughly at $t \cong$ $R_{A} / \gamma_{\mathrm{cm}}=R_{A} \cdot 2 m_{N} / \sqrt{s_{N N}}$, i.e. at about $2.5,1.5$, and $0.8 \mathrm{fm} / c$, respectively.

The primary energetic nucleon-nucleon collisions still yield a strong momentum anisotropy for particles inside the cell. During this entire highest energy density phase the widths of the velocity distributions in the longitudinal $(z-)$ direction, $\sigma_{z}$, are much larger then the widths in the transverse $(x-, y-)$ directions, $\sigma_{x}$ and $\sigma_{y}$, for each hadron species, respectively. However, the individual anisotropies inside the central cell gradually disappear with increasing time and the widths, $\sigma_{z}$ and $\sigma_{x}=\sigma_{y}$, become nearly equal. The shape of the momentum distributions, $d N_{i} / d p_{z}$ and $d N_{i} / d p_{x}, d N_{i} / d p_{y}$, for different particle species " $i$ ', which are quite different in the initial reaction 
stage, become approximately equal at $t \geq t_{\mathrm{eq}}$. For all three bombarding energies we have found $t_{\mathrm{eq}} \cong 10$ $\mathrm{fm} / c$ for the central cell. The isotropy of the hadron distributions in the central cell appears at $t \geq 10$ $\mathrm{fm} / c$ as a result of the many hadron rescatterings and due to the rapid escape of fast and/or nonscattered particles from the cell. The isotropy of the momentum distributions for all hadron species is one necessary prerequisite for LTE.

The largest values of $\varepsilon$ and $\rho_{B}$ in the central reaction zone are reached earlier, at $t<t_{\text {eq }}$. During this non-equilibrium stage the matter in the cell consists of a large fraction of non-formed particles (produced mainly in string excitations). These particles are not thermalized and exhibit a strong momentum anisotropy. Hence, the physical interpretation of this non-equilibrium early hadron-string phase of the reaction should - in the present approach - not be carried out in a statistical mechanics model (SM), although it has become popular to analyze string decays in terms of thermal/statistical model: Becattini has demonstrated that the fragmentation even of $e^{+} e^{-}$events leads to roughly statistical distributions with, however, nonequilibrium strangeness abundances [28-30].

The values of $\varepsilon$ and $\rho_{B}$ in the central cell found in the UrQMD simulations for AGS, "CERN" and SPS reactions at $t \geq t_{\mathrm{eq}}$ are shown in Fig. 1(a). For $t=10 \mathrm{fm} / c$ the fraction of non-formed particles is 20-30\% and goes to "zero" rapidly. The hadronresonance phase in this central cell at $t \geq 10 \mathrm{fm} / c$ and its thermal and chemical equilibration is the subject of the present study. Fig. 1(a) shows that the energy per baryon in the cell, $\varepsilon / \rho_{B}$, is approximately equal to 2,3 and $5 \mathrm{GeV}$ for AGS, "CERN", and SPS, respectively. It decreases by $10-20 \%$ during the expansion stage $t_{\mathrm{eq}}-t_{f}$ (see below).

The total number of particles in the cell becomes small at "large" $t$ and the system gradually approaches its "freeze-out" (collisions cease) stage. The UrQMD-cell calculations are stopped at $t=t_{f} \approx$ $20 \mathrm{fm} / c$. The analysis of the LTE-issues is restricted to this time interval, $t_{\mathrm{eq}} \leq t \leq t_{f}$. The values of $t_{f}$ are estimated by two different criteria: $\varepsilon(t)=\varepsilon_{f}=100$ $\mathrm{MeV} / \mathrm{fm}^{3}$ and $\rho_{\text {tot }}(t)=\rho_{\text {tot }}^{f}=0.5 \rho_{0}\left(\rho_{\text {tot }}\right.$ is the total particle number density, $\rho_{0}=0.16 \mathrm{fm}^{-3}$ is the ground state nuclear density). The first (second) criterion leads to the following estimates of $t_{f}: 18$
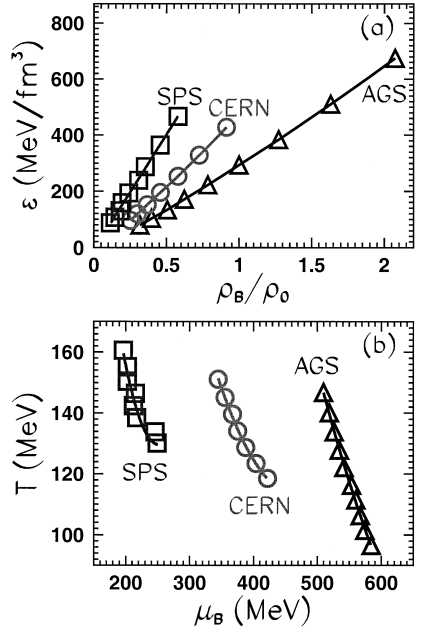

Fig. 1. (a): The evolution of baryon density, $\rho_{B} / \rho_{0}$, and energy density, $\varepsilon$, predicted by UrQMD model in the central cell of $\mathrm{Au}+\mathrm{Au}$ collisions at $10.7 \mathrm{AGeV}$ (AGS), $\mathrm{Pb}+\mathrm{Pb}$ collisions at 40 $\mathrm{AGeV}$ ('CERN') and $160 \mathrm{AGeV}$ (SPS) at times $t_{i}-t_{f}$, where $t_{i}=10 \mathrm{fm} / c$, and $t_{f}=18$ (AGS), 16 ("CERN") and $18 \mathrm{fm} / c$ (SPS). Within this time interval the energy per baryon is nearly constant for all three energies: $\varepsilon / \rho_{B}=2$ (AGS), 3 ("CERN"), 5 AGeV (SPS). It decreases by 10-20\% at $t=t_{f}$. (b): The same as (a), but for the $\left(\mu_{B}, T\right)$-plane. The values of baryon chemical potential, $\mu_{B}$, and temperature, $T$, are obtained by SM fit.

(17), 16 (16) and 18 (20) fm/c for AGS, "CERN", and SPS reactions, respectively. The "initial" nonequilibrium stage, $t<t_{\mathrm{eq}}=10 \mathrm{fm} / c$, with high energy density, has been studied in $[31,16]$ and more recently in [32-34]. The "final" kinetic stage $t>t_{f}$ of particle freeze-out and subsequent resonance decays is to be studied separately.

\section{Comparison with SM of ideal hadron-reso- nance gas}

UrQMD events of A + A collisions at AGS, "CERN" and SPS are analyzed below. The following procedure is applied. The ensemble average of the energy density, $\varepsilon$, net baryon density, $\rho_{B}$, and net strangeness density, $\rho_{S}$, in the central cell are calculated for $t_{\mathrm{eq}} \leq t \leq t_{f}$. The contributions of both formed and not yet formed particles are included. Then the SM of the ideal hadron-resonance gas is taken with the same 55 baryon and 32 meson species and their antistates as in the UrQMD model. Thus, 
the UrQMD string contributions to $\varepsilon, \rho_{B}, \rho_{S}$ are contained in those of the not-yet-formed particles. Therefore, string degrees of freedom should not be included in the SM.

The thermodynamical parameters of the SM system - temperature $T$, baryochemical potential $\mu_{B}$ and strange chemical potential $\mu_{S}$ - are extracted at each time-step of the UrQMD evolution. For each particle species " $i$ '" we assume the Boltzmann distribution function

$f_{i}=\exp \left(\frac{\mu_{i}-E_{i}}{T}\right)$,

where $E_{i}=\left(p^{2}+m_{i}^{2}\right)^{1 / 2}$ is the particle energy and $\mu_{i}$ is the chemical potential presented as

$\mu_{i}=b_{i} \mu_{B}+s_{i} \mu_{S}$,

with possible particle species " $i$ ', charges $\left(b_{i}=0, \pm\right.$ $\left.1 ; s_{i}=0, \mp 1, \mp 2, \mp 3\right)$. The electrochemical potential considered in Refs. [35,36] is neglected.

The UrQMD values of $\varepsilon, \rho_{B}$ and $\rho_{S}$ serve as input to the SM, where these values are inserted in the 1.h.s. of the following equations:

$\varepsilon=\sum_{i} \varepsilon_{i}, \quad \rho_{B}=\sum_{i} b_{i} n_{i}, \quad \rho_{S}=\sum_{i} s_{i} n_{i}$.

Here the particle number densities, $n_{i}$, and energy densities, $\varepsilon_{i}$, on the r.h.s. of Eq. (3) are calculated as:

$n_{i}=\frac{d_{i}}{2 \pi^{2} \hbar^{3}} \int_{0}^{\infty} p^{2} d p f_{i}$,

$\varepsilon_{i}=\frac{d_{i}}{2 \pi^{2} \hbar^{3}} \int_{0}^{\infty} p^{2} d p E_{i} f_{i}$.

Here $d_{i}$ is the spin-isospin degeneracy factor of the hadron species " $i$ '. The sums in Eq. (3) are taken over all hadron-resonance species presented in the UrQMD. Quantum statistical effects are not included in the present analysis. Note, however, that these effects are small for the $T$ and $\mu_{i}$ values considered below. The only visible difference (about 10\%) between quantum and classical descriptions can be observed in the pion yields.

The time evolution of the $T$ and $\mu_{B}$ values in the cell is shown in Fig. 1(b). It is also presented in the $\left(\rho_{B}, T\right)$-plane in Fig. 2(a).

The hadron pressure is given in the SM by

$P\left(T, \mu_{B}, \mu_{S}\right)=\sum_{i} \frac{d_{i}}{2 \pi^{2} \hbar^{3}} \int_{0}^{\infty} p^{2} d p \frac{p^{2}}{3 E_{i}} f_{i}$.


Fig. 2. (a): The evolution of baryon density, $\rho_{B} / \rho_{0}$, and SM temperature, $T$, in the central cells of the three reactions in question at times $t_{i}-t_{f}$. (b): The same as (a), but for the ( $\varepsilon, P)$-plane. The ratio $P(\varepsilon) / \varepsilon$ is nearly constant for all reactions: 0.12 (AGS), 0.14 ("CERN") and 0.16 (SPS)

LTE of the hadron matter in the cell manifests itself in the isotropy of the microscopic pressure components [26,37]:

$P_{\{x, y, z\}}^{\text {mic }}=\frac{1}{V} \sum_{h} \frac{p_{h\{x, y, z\}}^{2}}{3\left(m_{h}^{2}+p_{h}^{2}\right)^{1 / 2}}$,

where $V$ is the volume of the cell, $p_{h}$ represents the particle momentum and the sum in Eq. (7) is taken over all hadrons $h$ in the cell. A strong difference between longitudinal, $P_{z}^{\text {mic }}$, and transverse, $P_{x}^{\text {mic }}=$ $P_{y}^{\text {mic }}$, components of the microscopic pressure tensor is observed at the initial anisotropic non-equilibrium stage of $\mathrm{A}+\mathrm{A}$ reactions. At $t=t_{\mathrm{eq}}$ the microscopic pressure in the cell becomes isotropic and approximately equal to the ideal gas SM pressure (6):

$P^{\text {mic }} \equiv \sum_{k=x, y, z} P_{k}^{\text {mic }} \cong P\left(T, \mu_{B}, \mu_{S}\right)$

This leads us to conclude that the hadron-resonance matter in the cell - during the time interval $\left(t_{\mathrm{eq}}, t_{f}\right)-$ can be described in terms of the EoS (6), which in turn corresponds to the grand canonical SM equilib- 
rium. This gives a justification for using the hydrodynamical evolution approach. The ratio of the pressure to the energy density shows a measure of hydrodynamical effects in the system (see, e.g. [38]). This ratio in the cell remains approximately constant during the whole expansion stage $\left(t_{\mathrm{eq}}, t_{f}\right)$ and increases slightly with $E_{\text {lab }}$ [see Fig. 2(b)]:

$P / \varepsilon \cong 0.12$ (AGS), 0.14 ("CERN") ,

0.16 (SPS) .

The entropy density $s=s\left(T, \mu_{B}, \mu_{S}\right)$ can be calculated from the thermodynamical identity

$s \equiv \frac{1}{T}\left(\varepsilon+P-\mu_{B} \rho_{B}-\mu_{S} \rho_{S}\right)$.

The entropy per baryon $s / \rho_{B}$ in the cell for $t_{\mathrm{eq}} \leq t$ $\leq t_{f}$ is nearly constant and increases with $E_{\text {lab }}$ :

$s / \rho_{B} \cong 12(\mathrm{AGS}), \quad 20$ ("CERN'), 38 (SPS) .

Therefore, the hadron-resonance matter evolution in the cell is similar to an ideal hydrodynamic expansion during the time interval $\left(t_{\mathrm{eq}}, t_{f}\right)$.

\section{Non-equilibrium effects}

The results of the previous sections support a LTE picture in the central cell for $t_{\mathrm{eq}} \leq t \leq t_{f}$. It is formulated in terms of the ideal gas thermodynamical functions with their hydrodynamic-like evolution. Now we turn to the study of spectra for individual particle species. In Figs. 3(a)-(c) we show the comparison of the UrQMD particle spectra in the cell at $t=t_{\mathrm{eq}}=10 \mathrm{fm} / c$ with ideal gas Boltzmann distributions

$$
\begin{aligned}
\frac{d^{3} N}{d^{3} p} & =\frac{d N}{4 \pi p E_{i} d E_{i}} \\
& =\frac{V d_{i}}{(2 \pi \hbar)^{3}} \exp \left(\frac{B_{i} \mu_{B}+S_{i} \mu_{S}}{T}\right) \exp \left(-\frac{E_{i}}{T}\right) .
\end{aligned}
$$

The parameters $T, \mu_{B}, \mu_{S}$ in Eq. (12) are extracted by means of the SM from Eqs. (1)-(5). For AGS reactions [Fig. 3(a)] the shape and absolute normalization of the UrQMD spectra are rather close to those given

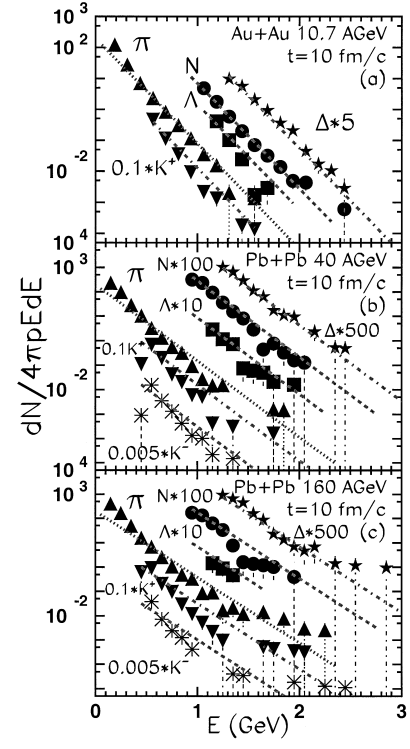

Fig. 3. Energy spectra of $N(\mathbf{O}), \Lambda(\boldsymbol{\square}), \pi(\boldsymbol{\Delta}), K^{+}(\boldsymbol{\nabla}), K^{-}$ (*) and $\Delta(\star)$ in central $125 \mathrm{fm}^{3}$ cell of $\mathrm{A}+\mathrm{A}$ collisions at 10.7 $\mathrm{A} \cdot \mathrm{GeV}(\mathrm{a}), 40 \mathrm{AGeV}$ (b) and $160 \mathrm{AGeV}$ (c) at $t=10 \mathrm{fm} / c$ are fitted by Boltzmann distributions, Eq. (12) (lines), using the $T, \mu_{B}, \mu_{S}$ parameters given in Table 1.

by Eq. (12). The different hadron species exhibit thermal equilibrium (the same inverse slope "temperature") and chemical equilibrium as it is required by the SM. When the collision energy increases we observe, however, significant deviations from the SM picture. The meson spectra in the central cell at "CERN" and SPS show systematically lower inverse slope 'temperatures' in comparison to the $T$ values found from the SM. This is not the result of a collective flow effect (which is negligible inside the central cell). The most dramatic non-equilibrium effect seen in Fig. 3(c) takes place for pions at SPS collisions. The pion inverse slope temperature is smaller than the $T$ values extracted from the ideal gas SM. However, the pion number density in the cell is larger than the SM result by a factor of two! The observed strong chemical non-equilibrium effects for the pion sub-system requires the presence of large positive pion chemical potential, $\mu_{\pi}$, instead of $\mu_{\pi}=0$ as in an equilibrium SM treatment.

Another important point of these results is the incomplete thermal equilibrium in the cell. Let us look again at the pions: At each time step in the 
Table 1

Temperature, $T$, baryon chemical potential, $\mu_{B}$, and strangeness chemical potential, $\mu_{S}$, obtained in the statistical model of the ideal hadron gas for the central cell of $\mathrm{A}+\mathrm{A}$ collisions at AGS, "CERN", and SPS energies at $t=10 \mathrm{fm} / c$

\begin{tabular}{clll}
\hline Energy, AGeV & $T, \mathrm{MeV}$ & $\mu_{B}, \mathrm{MeV}$ & $\mu_{S}, \mathrm{MeV}$ \\
\hline 10.7 & 147 & 510 & 129 \\
40.0 & 151 & 345 & 74 \\
160.0 & 161 & 197 & 36.8 \\
\hline
\end{tabular}

evolution of the system an essential fraction of the pions escapes straight from resonance and string decays, without rescatterings. These pions had no chance to thermalize and reach a Boltzmann shape spectrum through elastic rescatterings. The typical number of elastic rescatterings per each newly produced pion is roughly 1.5 in the dense hadron-resonance gas.

\section{Conclusions}

The equilibration of hadronic matter produced in the central zone of heavy ion collisions at projectile energies from 10.7 to $160 \mathrm{AGeV}$ has been studied in the microscopic UrQMD model. The following conclusions may be drawn.

1. There is a quasi-equilibrium stage of hadronresonance matter in the central cell of volume $V=$ $125 \mathrm{fm}^{3}$ during the time interval from $t=t_{\mathrm{eq}} \cong 10$ $\mathrm{fm} / c$ up to $t=t_{f} \cong 18 \pm 2 \mathrm{fm} / c$. Collective flow effects inside the central cell are negligible in comparison to the thermal motion during this quasi-equilibrium stage. The energy per baryon during this stage is approximately constant and equals 2, 3 and 5 GeV for AGS, "CERN', and SPS energies, respectively. It decreases by 10-20\% during the expansion stage $t_{\mathrm{eq}}-t_{f}$.

2. The macroscopic variables in the central cell can be described in terms of SM model during time interval $\left(t_{\mathrm{eq}}, t_{f}\right)$. The ratio of the pressure to the energy density is approximately constant and equals 0.12 (AGS), 0.14 ("CERN") and 0.16 (SPS). The time evolution of thermodynamical variables is similar to an ideal hydrodynamic model. It corresponds to a constant $s / \rho_{B}(S / A)$-ratio, which equals $s / \rho_{B}$ $\cong 12$ (AGS), 20 ('CERN') and 38 (SPS).
3. The UrQMD model predicts a local quasi-equilibrium state of a hadron-resonance gas. This appears to be close to the SM equilibrium at AGS energies. Here the spectra for all particle species are in a good agreement with the SM equilibrium results. At higher collision energies, however, we observe a significant deviation of the UrQMD-cell results from the SM equilibrium. These deviations appear at energies per baryon, $\varepsilon / \rho_{B}$, larger than $2 \mathrm{GeV}$ and increase with rising $E_{\text {lab }}$. At high energy per baryon values, $\varepsilon / \rho_{B} \approx 5 \mathrm{GeV}$, typical for the quasi-equilibrium stage in SPS collisions, a strong enhancement of a factor of 2 is observed in the pion number density, which points to the necessity of including finite pion chemical potentials to the SM analysis.

\section{Acknowledgements}

Fruitful discussions with K. Bugaev, L. Csernai, M. Gaździcki, I. Mishustin, St. Mrówczyński, L. Neise and L. Satarov are greatfully acknowledged. This work was supported by the A. v. Humboldt Stiftung, GSI, DFG, BMBF, Graduiertenkolleg " Experimentelle und Theoretische Schwerionenphysik" and the Buchmann Stiftung.

\section{References}

[1] E. Fermi, Prog. Theor. Phys. 5 (1950) 570; Phys. Rev. 81 (1951) 683.

[2] I.Ja. Pomeranchuk, Doklady Akad. Nauk SSSR 78 (1951) 889.

[3] R. Hagedorn, Suppl. Nuovo Cim. 3 (1965) 147; R. Hagedorn, J. Rafelski, Phys. Lett. B 97 (1980) 136.

[4] J. Cleymans, H. Satz, Z. Phys. C 57 (1993) 135; J. Cleymans, K. Redlich, H. Satz, E. Suhonen, Z. Phys. C 58 (1993) 347.

[5] P. Braun-Munzinger, J. Stachel, J.P. Wessel, N. Xu, Phys. Lett. B 344 (1995) 43; B 365 (1996) 1; J. Stachel, Nucl. Phys. A 610 (1996) 509c.

[6] J. Sollfrank, J. Phys. G 23 (1997) 1903.

[7] R.A. Ritchie, M.I. Gorenstein, H.G. Miller, Z. Phys. C 75 (1997) 2002; G.D. Yen, M.I. Gorenstein, W. Greiner, S.N. Yang, Phys. Rev. C 56 (1997) 2210; G.D. Yen, M.I. Gorenstein, H. Stöcker, S.N. Yang, W. Greiner, J. Phys. G 24 (1998) 1777; G. D Yen, M.I. Gorenstein, Phys. Rev. C 59 (1999) 2788.

[8] L.D. Landau, Izv. Akad. Nauk SSSR 17 (1953) 51; S.Z. Belenkij, L.D. Landau, Suppl. Nuovo Cim. 3 (1956) 15.

[9] J.D. Bjorken, Phys. Rev. D 27 (1983) 140. 
[10] A.A. Amsden, A.S. Goldhaber, F.H. Harlow, J.R. Nix, Phys. Rev. C 17 (1978) 2080; L.P. Csernai et al., Phys. Rev. C 26 (1982) 149; R.B. Clare, D. Strottman, Phys. Rep. 141 (1986) 177; H.W. Barz, B. Kämpfer, L.P. Csernai, B. Lukacs, Nucl. Phys. A 465 (1987) 743; H.W. Barz, B. Kämpfer, Phys. Lett. B 206 (1988) 399.

[11] S. Bernard, J.A. Maruhn, W. Greiner, D.H. Rischke, Nucl. Phys. A 605 (1996) 566.

[12] B.R. Schlei, U. Ornik, M. Plumer, D. Strottman, R.M. Weiner, Phys. Lett. B 376 (1996) 212.

[13] J. Brachmann, Nucl. Phys. A 619 (1997) 391.

[14] E. Shuryak, Sov. J. Nucl. Phys. 16 (1972) 395.

[15] J. Hofmann, H. Stöcker, U. Heinz, W. Scheid, W. Greiner, Phys. Rev. Lett. 36 (1976) 88.

[16] K. Geiger, Phys. Rep. 258 (1995) 237.

[17] U. Heinz, J. Phys. G 25 (1999) 263; Nucl. Phys. A 638 (1998) 357c; E. Schnedermann, J. Sollfrank, U. Heinz, Phys. Rev. C 48 (1993) 2462.

[18] S.A. Bass et al., Prog. Part. Nucl. Phys. 41 (1998) 225; M. Bleicher et al., J. Phys. G 25 (1999), in press.

[19] N.S. Amelin, L.V. Bravina, L.I. Sarycheva, L.N. Smirnova, Sov. J. Nucl. Phys. 51 (1990) 1093; N.S. Amelin et al., Phys. Rev. C 47 (1993) 2299.

[20] H. Sorge, H. Stöcker, W. Greiner, Ann. of Phys. 192 (1989) 266.

[21] Y. Pang, T.J. Schagel, S.H. Kahana, Phys. Rev. Lett. 68 (1992) 2743.
[22] Bao-An Li, Che Ming Ko, Phys. Rev. C 52 (1995) 2037; 53 (1996) 22.

[23] M. Bleicher, Phys. Lett. B 435 (1998) 9.

[24] M. Belkacem, Phys. Rev. C 58 (1998) 1727.

[25] L.V. Bravina, Phys. Lett. B 434 (1998) 379.

[26] M. Berenguer, J. Phys. G 18 (1992) 655.

[27] Status and Future Programme of the NA49 Experiment, preprint CERN/SPSC 98-4.

[28] F. Becattini, J. Phys. G 25 (1999) 287.

[29] S.A. Bass, Phys. Rev. Lett. 81 (1998) 4092.

[30] J. Sollfrank, U. Heinz, H. Sorge, N. Xu, J. Phys. G 25 (1999) 363.

[31] H. Sorge, A.v. Keitz, R. Mattiello, H. Stöcker, W. Greiner, Z. Phys. C 47 (1990) 629.

[32] K. Geiger, B. Müller, Heavy Ion Phys. 7 (1998) 207.

[33] H. Weber, Nucl. Phys. A 642 (1998) 121.

[34] H. Weber, Phys. Lett. B 442 (1998) 443.

[35] M.I. Gorenstein, S.N. Yang, Phys. Rev. C 44 (1991) 2875; M.I. Gorenstein, H.G. Miller, R.M. Quick, S.N. Yang, Phys. Rev. C 50 (1994) 2232; M.I. Gorenstein, H.G. Miller, Phys. Rev. C 55 (1997) 2002.

[36] J. Cleymans, D. Elliot, H. Satz, R.L. Thews, Z. Phys. C 74 (1997) 319.

[37] H. Sorge, Phys. Rev. Lett. 78 (1997) 2309; Phys. Lett. B 402 (1997) 251.

[38] C.M. Hung, E. Shuryak, Phys. Rev. Lett. 75 (1995) 4003; Phys. Rev. C 56 (1997) 453. 